1 Body Perception in Chimpanzees (Pan troglodytes): The Effect of Body Structure Changes

Jie Gao

Kyoto University; Japan Society for the Promotion of Science

Masaki Tomonaga

Kyoto University
6

Gao, J., \& Tomonaga, M. (2020). Body perception in chimpanzees (Pan troglodytes): The effect of body structure changes. Journal of Comparative Psychology. Advance online (1) publication. https://doi.org/10.1037/com0000214

[This is the final accepted manuscript.] 
Author Note

Jie Gao, Primate Research Institute, Graduate School of Science, Kyoto University;

24 Japan Society for the Promotion of Science; Masaki Tomonaga, Primate Research Institute,

25 Kyoto University.

This study was supported by the Ministry of Education, Culture, Sports, Science and

Technology of Japan (MEXT)/Japan Society for the Promotion of Science (JSPS) KAKENHI

Core CCSN, National BioResource Project-Great Ape Information Network, and MEXT

Scholarship (\#152121). The authors thank Drs. T. Matsuzawa, I. Adachi, M. Hayashi, Y. Hattori

and T. Takashima, and other colleagues of Language and Intelligence Section. Thanks are also due to staff of the Center for Human Evolution Modeling Research at the Primate Research

Institute for taking care of the chimpanzees. We also thank Kumamoto Sanctuary for providing chimpanzee photos. 
Abstract

Chimpanzees have been found to show the inversion effect to visual stimuli of intact chimpanzee bodies, suggesting that they have a specific way of body processing. In this study, we examined how changes of body structures affect the inversion effect to reveal the properties of their body processing. We focused on two aspects of body structures: the first-order relations (i.e. body part arrangements), and body proportions. The experimental conditions had stimuli of chimpanzee bodies with scrambled first-order relations in Experiment 1, and chimpanzee bodies with distorted body proportions in Experiment 2. Both experiments had a control condition consisting of stimuli of intact chimpanzee bodies. Seven chimpanzees participated in matching-to-sample tasks on touch screens. In each trial, the stimuli were chimpanzee bodies with the same kind of manipulation of body structures and were either all upright or all inverted.

54 We compared their performances in the upright and inverted trials to examine the inversion effect. The chimpanzees did not show any inversion effect to scrambled bodies, but showed the inversion effect to distorted bodies and to intact bodies. The results suggest that chimpanzees' configural body processing relies on correct first-order relations, and distorted body proportions do not interfere with configural processing. It also implies that chimpanzees may have knowledge of the arrangement of body parts, but they may be less sensitive to body

60 proportions. This study could facilitate the understanding of the evolution of visual processing 61 of bodies, faces, and other objects. Keywords: body perception, inversion effect, configural processing, body structure, 


\section{Body Perception in Chimpanzees (Pan troglodytes): The Effect of Body Structure Changes}

This study aims to investigate how changes of body structures influence the body perception of chimpanzees, based on the knowledge of their processing of bodies with normal structures (Gao \& Tomonaga, 2018). We hope to reveal detailed mechanisms of chimpanzees' body processing by using stimuli of abnormal chimpanzee bodies, in order to understand the evolution of body processing and why bodies are processed differently from other objects. We also hope the study could shed light on chimpanzees' understanding of body structures to provide an evolutionary perspective on how objects are configured by animals.

Bodies are important social cues for animals. Chimpanzees, for example, can recognize other individuals based on bodies (Martinez \& Matsuzawa 2009; Matsuzawa, Tomonaga, \& Tanaka, 2006). They learn to use tools, in part, by viewing others' body actions (Matsuzawa, Humle, \& Sugiyama, 2011), and use various kinds of bodily gestures for social communication (Hobaiter \& Byrne, 2014). They use their own bodies for foraging, and other daily activities (Matsuzawa, Humle, \& Sugiyama, 2011). Behind these behaviours, there must be certain specific perceptions and understanding in chimpanzees' minds of their bodies and how bodies are structured. The understanding of body structures might facilitate discrimination between conspecifics and other species and, potentially, special care for individuals with disabilities (Hirata, Hirai, Nogami, Morimura, \& Udono, 2017; Matsumoto, Itoh, Inoue, \& Nakamura, 2016), which is vital for their living. Additionally, as humans' closest living relatives, chimpanzees' understanding of their body structures could shed light on the evolutionary path of humans' perception of bodies. 
In a previous study, Gao \& Tomonaga (2018) found that chimpanzees show the

86

87 inversion effect when visually recognizing bodies. They had better performances in recognizing chimpanzee bodies when they were upright compared to when they were inverted in a matching-to-sample task. The inversion effect suggests that chimpanzees use configural processing when recognizing bodies, that is, they use global cues of the body configuration instead of focusing on features of local parts of bodies. If they use cues of local features, the inversion would not deteriorate the performances very much. The inversion effect has been used as a robust index for configural processing (reviewed in Maurer, Le Grand, \& Mondloch, 2002). Chimpanzees did not show the inversion effect to house stimuli in the previous study, suggesting a unique way to process bodies that is different from what is used for other objects. The inversion effect has been found for body recognition in humans (e.g. Peelen \& Downing, 2007; Quinn, Lee, Pascalis, \& Slater, 2007; Reed, Stone, Bozowa, \& Tanaka, 2003; Reed, Stone, Grubb, \& McGoldrick, 2006; Yovel, Pelc, \& Lubetzky, 2010) and capuchin monkeys (Matsuno \& Fujita, 2018). The inversion effect and configural processing are not limited to bodies, actually. It has also been found for faces (e.g. humans: Carey, 1992; Diamond \& Carey, 1986; Yin, 1969; chimpanzees: Dahl, Rasch, Tomonaga, \& Adachi, 2013; Parr, Dove, \& Hopkins, 1998; Tomonaga, 1999; Tomonaga \& Imura, 2015), dogs, birds, and cars when the viewers were experts (Diamond \& Carey, 1986; Gauthier, Skudlarski, Gore, \& Anderson, 2000; Hagen \& Tanaka, 2019), and a series of artificial objects called "Greebles" after the viewers were trained to be experts (Gauthier \& Tarr, 1997; Gauthier, Williams, Tarr, \& Tanaka, 1998). It was concluded that the objects invoking the inversion effect (and configural processing) need 
106 to meet the following three criteria (Diamond \& Carey, 1986). 1) Each class of this kind of

107 stimuli need to share a common layout of elements. For example, all faces have two eyes above

108 a nose, which is above a mouth; all bodies have a head on top of the torso, and arms all connect

109 to the torso from the shoulder part. This spatial arrangement of distinguishing parts is called

110 the first-order relations. 2) The stimuli of each class are identified by the different space

111 between the first-order relational features. The spacing between the distinguishing features is

112 called the second-order relations. Humans can detect at least one minute different of the angle

113 of facial part placements (Haig, 1984), which makes it possible to distinguish faces where the

114 eyes, noses, and mouths are in similar shapes. 3) The viewers should be able to exploit the

115 second-order relation cues, that is, experience mediates the effect, such that dog experts showed

116 the inversion effect to dogs but the novices did not (Diamond \& Carey, 1986). The Greebles

117 are a series of objects that share the first-order relations and differ in the second-order relations,

118 and humans are able to develop configural processing after training to be experts (Gauthier \&

119 Tarr, 1997; Gauthier, Williams, Tarr, \& Tanaka, 1998).

120 Bodies, apparently, also fall in one of these classes that meet the above three criteria

121 and could invoke the inversion effect. It is necessary to dig deeper if we want to understand the

122 underlying mechanisms of chimpanzees' configural body processing. The inversion effect

123 suggests the use of configural processing, but if we consider only the inversion effect shown

124 to intact bodies, this by itself cannot reveal which aspect of configural processing is used

125 (Maurer, Le Grand, \& Mondloch, 2002). Maurer and colleagues suggested that in face

126 recognition, the configural processing has three aspects. The face inversion effect was 
127 diminished by scrambling facial parts (e.g. Bentin, Allison, Puce, Perez, \& McCarthy, 1996;

128 Tanaka \& Farah, 1993). This finding shows that the configural face processing relies on correct

129 arrangement of facial parts, i.e. the first-order relations of faces. The variation in second-order

130 relations are also important for invoking the configural processing. When all facial parts

131 remained exactly the same, and only the space between them changes in each face, the

132 inversion effect was found (Le Grand, Mondloch, Maurer, \& Brent, 2001). On the contrary,

133 when the spacing were the same and only facial features differ, there was no inversion effect

134 and humans tend to use featural cues. The third aspect is that faces are processed holistically.

135 Humans have better performance in naming the owner of a facial part when it is presented

136 within the whole face, compared to when presented alone (Tanaka \& Farah, 1993).

137 For body recognition, Reed and colleagues (2006) found that humans' configural

138 processing relies on correct first-order relations, which is shared in face recognition (reviewed

139 in Maurer, Le Grand, \& Mondloch, 2002). They tested body recognition in a detection task.

140 When they used human bodies with misaligned body parts, the inversion effect was gone.

141 Similar results were reported in other studies in human adults (Gliga \& Dehaene-Lambertz,

142 2005; Yovel, Pelc, \& Lubetzky, 2010), 3-month-old human infants (Gliga \& Dehaene-

143 Lambertz, 2005), and capuchin monkeys when they recognize human bodies (Matsuno \&

144 Fujita, 2018). Studies in human infants showed that 3.5-month-old infants could discriminate

145 between intact bodies and bodies with abnormal first-order relations, and between intact bodies

146 and bodies with abnormal body proportions. They could make both discriminations when

147 bodies were upright, but not when they were inverted (Zieber, Bhatt, Hayden, Kangas, Collins, 
148 \& Bada, 2010; Zieber, Kangas, Hock, \& Bhatt, 2015). This finding suggests that young infants

149 are sensitive to the first-order relations of bodies and body proportions. However, as they did

150 not test the inversion effect directly, it is not clear how configural processing works in human

151 infants, especially the aspect about body proportions.

In order to understand the evolution of body processing, detailed examination of body

processing of chimpanzees is necessary. We investigated whether chimpanzees have similar

154 properties in body processing with humans. At the same time, we compared the properties of

face processing and body processing. There are many aspects to investigate in chimpanzees'

configural body processing. We focused on two kinds of body structures in this study. Firstly,

we examined the function of the first-order relations of bodies in the configural processing. We

and with face processing in terms of first-order relations (Maurer, Le Grand, \& Mondloch,

160 2002). Secondly, inspired by the studies in human infants (Zieber, Bhatt, Hayden, Kangas,

161 Collins, \& Bada, 2010; Zieber, Kangas, Hock, \& Bhatt, 2015), we also studied the function of

162 body proportions in the body processing. Although it is under debate whether body proportion

163 is corresponding to the second-order relations of faces (Reed, Stone, Grubb, \& McGoldrick,

164 2006; Zieber, Bhatt, Hayden, Kangas, Collins, \& Bada, 2010), examining the possible effects

165 of changing this kind of body structure on body configural processing might provide indirect

166 information for the comparison of face and body recognition. We tested the inversion effect

167 using stimuli of chimpanzee bodies with abnormal first-order relations and abnormal body

168 proportions, respectively. The results might also help understand the body "prototype" in 
169

170

171

172

173

174

chimpanzees.

Gao \& Tomonaga (2018) tested the inversion effect in several different conditions.

There was not a condition where the body parts were misaligned in that study, but there were several where the head was missing or blurred. We found that the inversion effect was gone when the head was missing or blurred, but it was not affected when the other body parts except for the head were blurred. Therefore, we concluded that the head plays a more important role in invoking the inversion effect than the rest of the body parts. (Another important element is the body contour; see Gao \& Tomonaga, 2018 for details.) The primary role of the head in body processing is also supported by other studies in humans (e.g. Ramm, Cummins, \& Slaughter, 2010; Robbins \& Coltheart, 2012; Yovel, Pelc, \& Lubetzky, 2010). In this study, in the condition where first-order relations were manipulated, half of the stimuli had the head misplaced, and the other half did not. We additionally compared the results of the two kinds of stimuli apart from analyzing all data together in this condition, to examine whether the head contributes more than other body parts in the possible effect of body structure changes on the body processing.

Method

\section{Participants}

Seven chimpanzees participated in this study (6 chimpanzees in Experiment $1 ; 7$ chimpanzees in Experiment 2; Table 1). They are housed at the Primate Research Institute, Kyoto University (Inuyama, Aichi, Japan). They belong to two social groups composed of 12 individuals in total. They live in a large environment that consists of an outdoor compound 
$190\left(700 \mathrm{~m}^{2}\right)$ and attached indoor areas (Matsuzawa, Tomonaga, \& Tanaka, 2006). The animals

191 were not deprived of food or water during the study. The participants had experienced various

192 cognitive tasks with touchscreens, including acquisition of an artificial language, numerical

193 sequence learning, short-term memory tests, tests about visual attention and visual search, and

194 facial recognition, with sufficient experience of the matching-to-sample paradigm (e.g.

195 Tomonaga, 1999; Tomonaga \& Imura, 2015; Tomonaga, Itakura, \& Matsuzawa, 1993). The

196 method we use, touch screen tasks, prevents possible effect from doing the experiment with

197 humans manually, and increases the accuracy and consistency of the data. Only the seven

198 chimpanzees that we tested are familiar with touch screen and matching-to-sample tasks. The

199 small sample size is a limitation of the study, but we hope it can provide crucial information

200 for cognition of chimpanzees.

201

202 Table 1 General characteristics of the seven chimpanzees

\begin{tabular}{cccccc}
\hline Name & $\begin{array}{c}\text { GAIN ID } \\
\text { Number* }\end{array}$ & Sex & $\begin{array}{c}\text { Age (when the } \\
\text { study started })\end{array}$ & Kinship & Participated in \\
\hline Ai & 0434 & Female & 40 & Ayumu's mother & Experiments 1, 2 \\
Ayumu & 0608 & Male & 16 & $\begin{array}{c}\text { Ai's son; } \\
\text { Pal's paternal half- }\end{array}$ & Experiments 1, 2 \\
Chloe & 0441 & Female & 36 & Cleo's mother & Experiments 1, 2 \\
Cleo & 0609 & Female & 16 & Chloe's daughter & Experiments 1, 2 \\
Pan & 0440 & Female & 33 & Pal's mother & Experiment 2 \\
Pal & 0611 & Female & 16 & $\begin{array}{c}\text { Pan's daughter; } \\
\text { Ayumu's paternal }\end{array}$ & Experiments 1, 2 \\
\hline
\end{tabular}


half-sibling

$\begin{array}{lllll}\text { Pendesa } & 0095 & \text { Female } & 39 & \text { N.A. }\end{array}$

Note: *Identification number for each chimpanzee listed in the database of the Great

204 Ape Information Network (GAIN); https://shigen.nig.ac.jp/gain/

205

The daily care and use of the chimpanzees adhered to the Third Version of Guidelines

207 for the Care and Use of Laboratory Primates of the Primate Research Institute, Kyoto

208 University. The research proposal was approved by the Animal Research Committee of Kyoto

209 University and by the Animal Welfare and Animal Care Committee of the Primate Research

210 Institute of Kyoto University. All procedures adhered to the Japanese Act on the Welfare and

211 Management of Animals.

212 Apparatus

213 The participants sat in the experimental booth and performed tasks on a touchscreen

214 with a 15 -inch LCD display $(1,024 \times 768$ pixels, 1 pixel $=0.297 \mathrm{~mm})$. During the experiments,

215 they could move freely, but they would always sit in front of the screen about $40 \mathrm{~cm}$ away. The

216 chimpanzees kept their postures natural and relaxed, and their heads were always upright. The

217 screens were not tilted significantly in any direction (Figure 1A). When they made a correct

218 choice, the feeder delivered a piece of apple or raisin via a tube automatically to a food tray

219 placed at the bottom of the display with a chime sound. If they made a wrong choice, no food

220 was provided, and an error buzzer sounded. The experiment programs were written and

221 operated with Microsoft Visual Basic 2010 software (Microsoft Corp.; Redmond, WA, USA). 

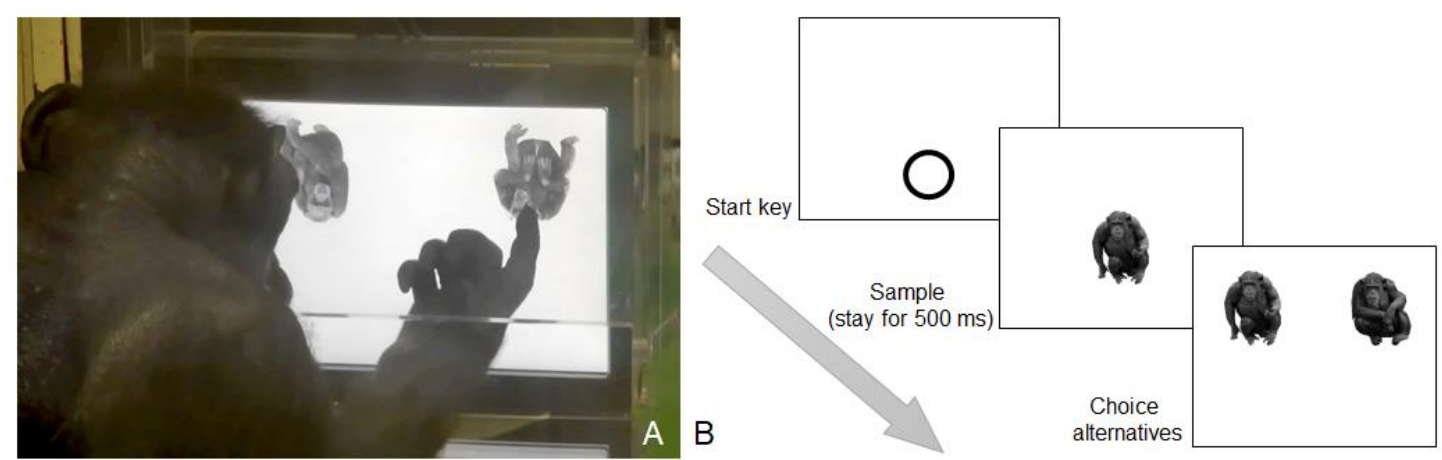

Ai is doing the task with the stimuli of two inverted chimpanzee bodies. B) In each trial of the experiment, a start key appeared on the screen, then a sample stimulus appeared after the participants touched the start key; the sample stayed for $500 \mathrm{~ms}$, and disappeared upon touching, and choice options appeared without any delay.

\section{Stimuli}

In Experiment 1, there were two conditions: intact body condition and scrambled body the stimuli was about $400 \mathrm{px} \times 400 \mathrm{px}$. For each pair, they showed the same individual, with whom the participants were not familiar, with the same kind of manipulations (e.g. both have their legs and arms swapped) but with different postures. Each pair had the same individual, so their heads were the same. The angles, shading and other properties of the faces were similar. 
241 Each trial used one pair (two pictures). Randomly, one picture would serve as a sample. Each

242 pair of pictures had the same orientation (upright or inverted), so, in each trial, all stimuli were

243 in the same orientation. The head and torso determined the orientation. In the upright

244 orientation, the head and torso of each stimulus were both presented upright. In the inverted

245 orientation, the head and torso of each stimulus were both inverted. The correct stimuli and

246 positions were counterbalanced. All stimuli were black and white and balanced in luminance.

247 In the intact body condition of Experiment 1, all stimuli were intact chimpanzees

248 (Figure 2). In the scrambled body condition of Experiment 1, all stimuli were chimpanzee

249 bodies that were scrambled in terms of first-order relations. There were ten kinds of

250 manipulations. Two upright pairs and their inverted versions adopted each kind of manipulation.

251 Among the ten kinds of manipulations, five included head position change: 1) head moved to

252 one arm, 2) head moved to one leg, 3) head and one leg swapped, 4) head and one arm swapped,

253 and 5) head moved to waist. The other five did not include head position change: 1) arms

254 moved to legs, 2) legs moved to arms, 3) one arm moved to head, 4) one leg moved to head,

255 and 5) legs and arms swapped.

256

In the intact body condition of Experiment 2, all stimuli were intact chimpanzees

257 (Figure 2). Small blurring parts in joints in the stimuli inevitably occurred during cutting body

258 parts and moving them in making the manipulated chimpanzee bodies using computer software.

259 We cut and moved certain body parts to make a strange body, and after moving them, we

260 needed to blur the joints to balance the luminance of the existing part and the newly moved

261 part in order to make it natural. The local blurring parts might affect the results. Therefore, we 
deliberately made small blurring parts in the intact body pictures for better control to rule out

263 and very long limbs (2 times of the originals).
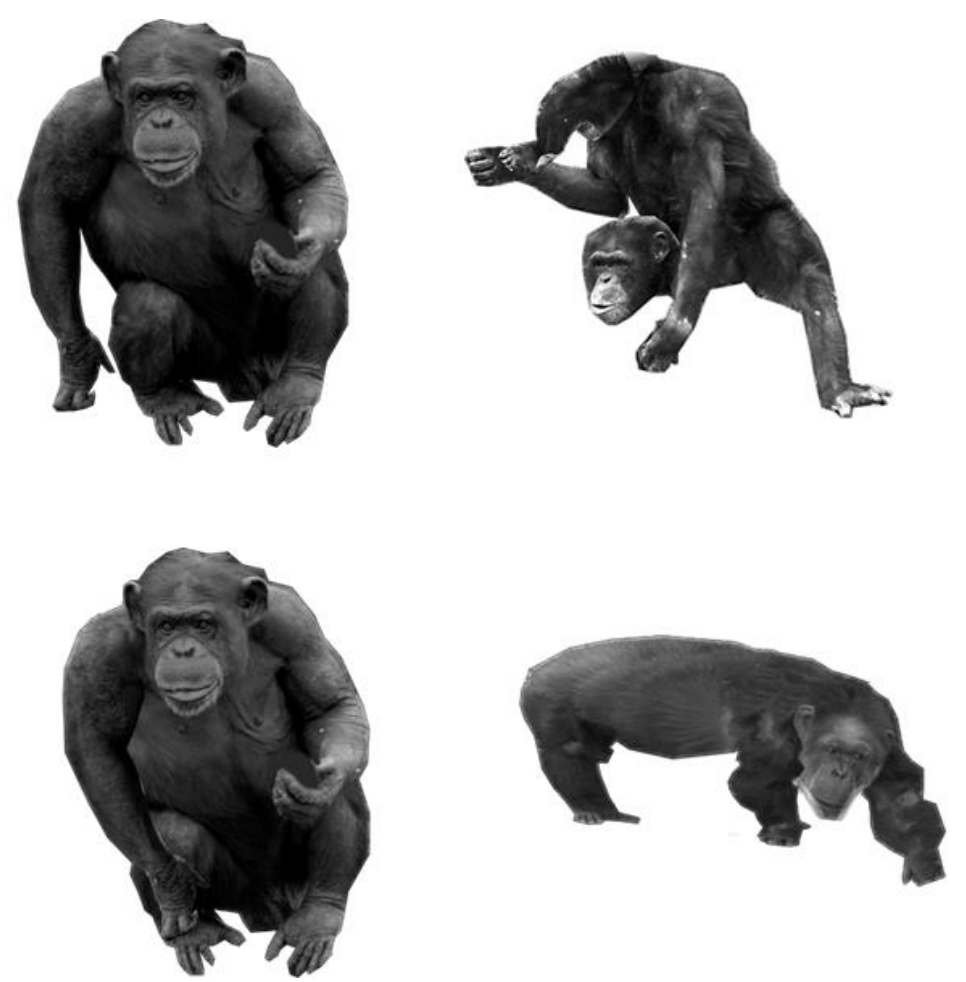

Figure 2. Sample Stimuli. Top three are sample stimuli for Experiment 1. Top left: a

sample for intact chimpanzee bodies. Top center: a sample for scrambled chimpanzee bodies

in terms of first-order relations with the change of the head position. Top right: a sample for scrambled chimpanzee bodies without the change of the head position. Bottom three are sample stimuli for Experiment 2. Bottom left: a sample for intact chimpanzee bodies with marks of 
276 with very long torso and very short limbs. Bottom right: a sample for distorted chimpanzee

277 bodies with very short torso and very long limbs.

\section{General procedure}

We used zero-delayed matching-to-sample tasks (Figure 1B). In each trial, a circle serving as the start key initially appeared at the bottom centre of the touchscreen against a white background. After chimpanzees touched the circle, a stimulus picture appeared in the centre of the screen. This served as the sample. After $500 \mathrm{~ms}$, after chimpanzees touched the sample, it would disappear, and, without any delay, two choice alternatives would appear side by side on the top of the screen. One of the two stimuli was the same as the sample, and they were a fixed

290 pair (the other stimulus was the same individual with the same kind of manipulation yet

291 different postures). If the participants touched the one that was the same as the sample, it was

292 a correct choice, and they would receive a piece of apple or raisin from the feeder with a chime

293 sound. If they chose the one that was not the same as the sample, it was a wrong choice, and a

294 buzzer would sound without any food reward followed by a timeout of $2000 \mathrm{~ms}$. The inter-trial interval was $1500 \mathrm{~ms}$. 
297 session had 40 trials. Twenty trials used upright stimuli (20 upright pairs), and the other half

298 used inverted stimuli (20 inverted pairs). The upright and inverted trials were randomly mixed.

\section{Data analyses}

300 We analysed accuracy and response-time data. We compared the accuracy and response

301 time between the upright and inverted trials. If either the error rate $(100 \%-$ accuracy $)$ or

302 response time was significantly higher in the inverted trials than in the upright trials, it showed

303 the inversion effect. We used the method of generalized linear mixed modelling (GLMM) in R

$304 \quad 3.4 .3$ (R Core Team; Vienna, Austria) using the lme4 package (Bates, Mächler, Bolker, \&

305 Walker, 2015). We calculated the accuracy of the 20 upright and 20 inverted trials on a session-

306 by-session basis. The distribution of the accuracy was binomial. We did the analyses for each

307 experiment separately. The model included as fixed effects condition (intact and

308 scrambled/distorted) and orientation (upright and inverted), and the interaction of condition

309 and orientation. The interaction was analyzed in order to examine whether the magnitude of

310 the inversion effect differs between the two conditions. The random effects were participant ID

311 and session number. To examine the potential effect of the manipulation of head in the

312 scrambled body condition of Experiment 1, we did another analysis using data from the

313 scrambled body condition only. We separated the data from the trials where the head position

314 was changed and the trials where the head position remained the same while only the positions

315 of other body parts were changed and marked them with the tag of 'sub-condition'. In these

316 analyses the model included sub-condition (with or without head position change) and

317 orientation (upright or inverted) as fixed effects, and the interaction between condition and 
318 orientation. The interaction could reveal a difference in the magnitude of the inversion effect.

319 The random effects were participant ID and session number. Response time refers to the

320 duration of time from the chimpanzee touching the sample to when s/he chose any of the

321 alternatives. Only the response times from correct trials were included in the analyses. We

322 calculated the average response time of all correct upright trials and all correct inverted trials

323 on a session-by-session basis. During the experiment, there would sometimes be unexpected

324 sounds from outside the experimental booths, and the chimpanzees were interrupted; therefore,

325 we initially discarded response times that were longer than the mean value plus 3 SDs. We then

326 calculated the average of the remaining data points and used them for further analyses. The

327 distribution of response time data was best fit with a gamma distribution. The analyses were

328 the same as for accuracy data except that the dependent variable was changed to response time,

329 and the distribution was changed to gamma distribution.

\section{Experiment 1}

\section{$331 \quad$ Results}

1. The intact body condition and the scrambled body condition

333 Accuracy

There was an inversion effect in the intact body condition $(p<.001)$ but not in the scrambled body condition $(p=.14)$. A significant interaction was found between condition and orientation $(p<.001)$.

In the intact body condition, the mean accuracy in the upright trials was $80.8 \pm 1.6 \%$, 
$[48.5,88.1]$ (Figure 3 ). In the scrambled body condition, the mean accuracy in the upright trials was $64.7 \pm 1.6 \%, 95 \% \mathrm{CI}[53.2,89.7]$, and the mean accuracy in the inverted trials was $67.1 \pm$ $1.4 \%, 95 \%$ CI $[56.7,91.2]$ (Figure 3). orientation: -0.16 , standard error: $0.11, z$ value $=-1.47, p=.14$; estimate of the fixed effect condition: -0.34 ; estimate of the intercept: 1.31 ).

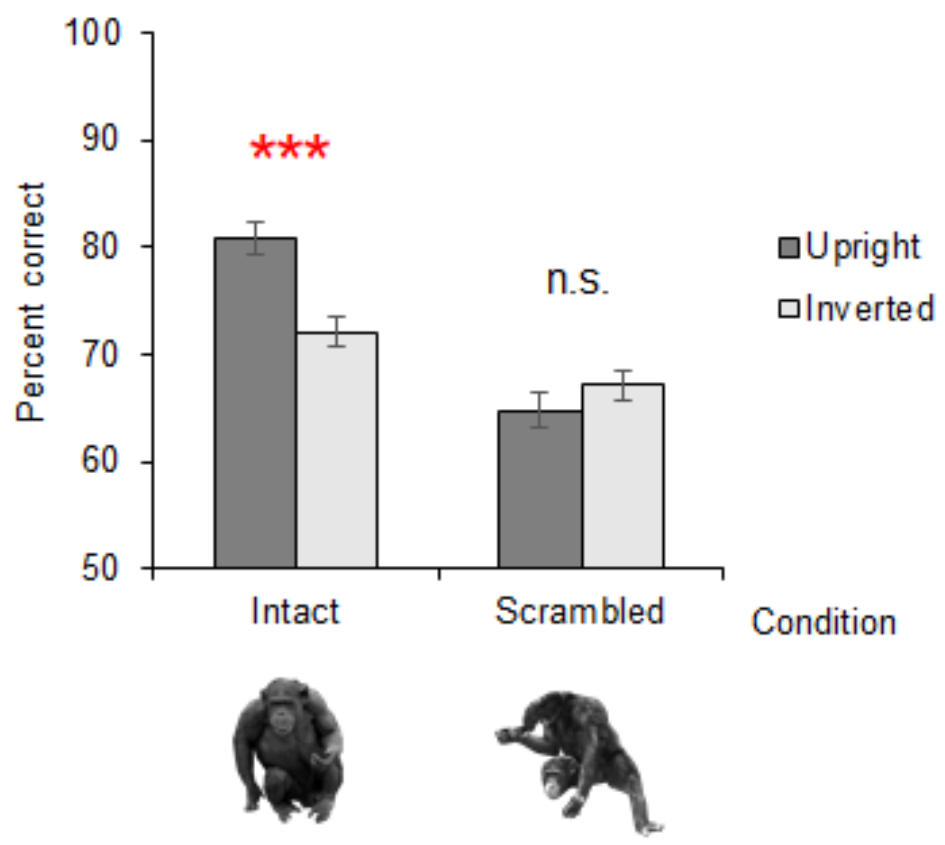


There was no inversion effect in the intact $(p=.81)$ or scrambled body condition ( $p$

$=.18)$. No interaction was found between condition and orientation $(p=.26)$. trials was $952 \pm 24 \mathrm{~ms}, 95 \% \mathrm{CI}[745,1275]$, and the mean response time in the inverted trials was $915 \pm 24 \mathrm{~ms}, 95 \%$ CI [723, 1209].

The GLMM analyses did not show significant differences between the upright and

362 inverted trials in the intact condition (estimate of the fixed effect orientation: $<0.001$, standard

363 error: $<0.001, t$ value $=-0.24, p=.81$; estimate of the fixed effect condition: $<0.001$; estimate

364 of the intercept: $<0.001$ ) or the scrambled condition (estimate of the fixed effect orientation:

$365<0.001$, standard error: $<0.001, t$ value $=1.34, p=.18$; estimate of the fixed effect condition:

$366<0.001$; estimate of the intercept: $<0.001)$. 


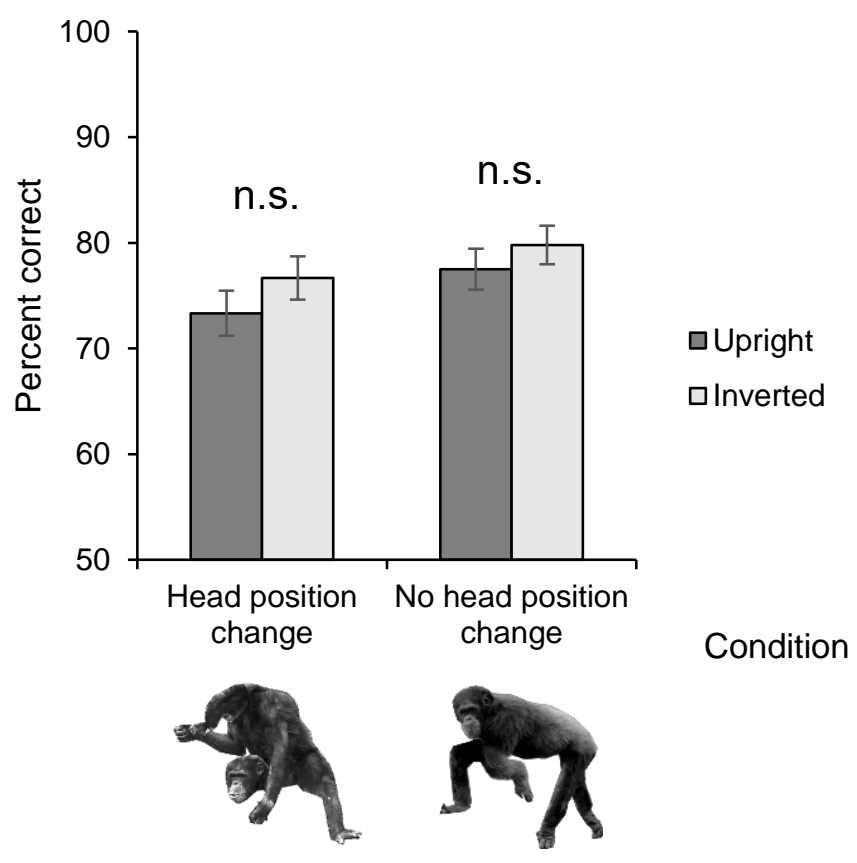

Figure 4. Chimpanzees' performances in the two sub-conditions in the scrambled

changes). Error bar: SEM; n.s.: not significant. position changes $(p=.39)$. No difference was found in accuracy between the two subconditions for the upright $(p=.13)$ or inverted trials $(p=.24)$. No interaction between subcondition and orientation was found $(p=.85)$. was $73.3 \pm 2.1 \%, 95 \% \mathrm{CI}[42.6,91.2]$, and the mean accuracy in the inverted trials was $76.7 \pm$ 
mean accuracy in the upright trials was $77.5 \pm 1.9 \%, 95 \%$ CI $[48.9,92.7]$, and the mean accuracy in the inverted trials was $79.8 \pm 1.8 \%, 95 \%$ CI $[52.8,93.5]$ (Figure 4). the performances in upright and inverted trials between the two sub-conditions, respectively. effect orientation: -0.14 , standard error: $0.16, z$ value $=-0.87, p=.39$; estimate of the intercept:

There was no difference in the performances between the two sub-conditions in the upright trials (when the reference of 'sub-condition' was set to the one with head position changes, estimate of the fixed effect sub-condition: 0.23 , standard error: $0.15, z$ value $=1.50$, $p=.13$ ) or the inverted trials (estimate of the fixed effect sub-condition: 0.18 , standard error:

$0.16, z$ value $=1.18, p=.24)$

Response time

There was no inversion effect from response-time data in the sub-conditions with ( $p$

$397=.20)$ or without head position changes $(p=.34)$. No difference in accuracy between the two 398 sub-conditions was found for the upright $(p=.14)$ or inverted trials $(p=.07)$. No interaction 399 between sub-condition and orientation was found $(p=.77)$. 
401 trials was $945 \pm 24 \mathrm{~ms}, 95 \%$ CI $[718,1345]$, and that in the inverted trials was $903 \pm 24 \mathrm{~ms}$,

$40295 \%$ CI $[696,1256]$. In the sub-condition without head position changes, the mean response

403 time in the upright trials was $996 \pm 35 \mathrm{~ms}, 95 \%$ CI [743, 1463], and that in the inverted trials

404 was $963 \pm 30 \mathrm{~ms}, 95 \%$ CI [728, 1386].

405 We first examined the inversion effect in the two sub-conditions, and then compared

406 the performances in upright and inverted trials between the two sub-conditions, respectively.

407 The GLMM analyses did not show significant differences between the upright and

408 inverted trials when the sub-condition was the one with head position changes (estimate of the

409 fixed effect orientation: $<0.001$, standard error: $<0.001, t$ value $=-1.30, p=.20$; estimate of

410 the intercept: $<0.001)$ or when the sub-condition was the one without head position changes

411 (estimate of the fixed effect orientation: $<0.001$, standard error: $<0.001, t$ value $=-0.95, p$

$412=.34$; estimate of the intercept: 0.001$)$.

413 There was no difference in the performances between the two sub-conditions in the

414 upright trials (when the reference of 'sub-condition' was set to the one with head position

415 changes: estimate of the fixed effect sub-condition: $<0.001$, standard error: $<0.001, t$ value $=$

$416-1.48, p=.14$ ) or the inverted trials (estimate of the fixed effect sub-condition: $<0.001$,

417 standard error: $<0.001, t$ value $=1.83, p=.07)$.

418 Discussion

No inversion effect was shown to scrambled chimpanzee bodies, suggesting that the

420 configural body processing in chimpanzees relies on correct first-order relations. This is

421 consistent with previous studies about body recognition in human adults (Gliga \& Dehaene- 
422 Lambertz, 2005; Reed, Stone, Grubb, \& McGoldrick, 2006; Yovel, Pelc, \& Lubetzky, 2010),

423 in 3-month-old infants (Gliga \& Dehaene-Lambertz, 2005), and in capuchin monkeys (to

424 human bodies; Matsuno \& Fujita, 2018). It is also the same as humans' face processing, where

425 correct first-order relations are necessary to invoke the inversion effect (e.g. Bentin, Allison,

426 Puce, Perez, \& McCarthy, 1996; Tanaka \& Farah, 1993). The results also indicate that

427 chimpanzees recognize correct first-order relations in building their body configuration.

428 No difference was found in the performances between the sub-conditions with or

429 without head position changes. This contradicts the predictions based on the findings in humans

430 (e.g. Ramm, Cummins, \& Slaughter, 2010; Robbins \& Coltheart, 2012; Yovel, Pelc, \&

431 Lubetzky, 2010) and chimpanzees (Gao \& Tomonaga, 2018). However, it might indicate that

432 the overall strange arrangement of body parts caught more attention in the chimpanzees.

433 In our previous study (Gao \& Tomonaga, 2018), we used individual discrimination to

434 test the inversion effect, that is, in each trial, the choice options were different individuals. It

435 could be possible that the body inversion effect we found was actually from the face inversion

436 effect, if there was no control condition to rule it out. We had a control condition consisting of

437 only chimpanzee faces, and the faces were in the same size as that of the faces in the bodies

438 we used (i.e. relatively small). There was no inversion effect, so actually the chimpanzees could

439 not use cues from only faces in recognizing bodies. However, a more direct evidence would be

440 more persuasive. In this study, we used posture discrimination, that is, both choice options in

441 one trial were the same individual (with the same head of course). The inversion effect shown

442 here directly supported that the inversion effects we found were not from the face inversion 
443 effect.

444 In order to understand further the properties of the configural body processing in 445 chimpanzees, in Experiment 2 we tested the effect of changes in another aspect of body 446 structures, body proportions, on the inversion effect.

447

\section{Experiment 2}

$448 \quad$ Results

449 Accuracy

450 There was an inversion effect in the intact $(p<.001)$ and distorted body conditions $(p$ $451=.03)$. No significant interaction between condition and orientation was found $(p=.11)$. In the intact body condition, the mean accuracy in the upright trials was $80.1 \pm 1.5 \%$, $45395 \% \mathrm{CI}[62.7,91.0]$, and the mean accuracy in the inverted trials was $72.3 \pm 1.5 \%, 95 \% \mathrm{CI}$ $454[50.9,87.2]$ (Figure 5). In the distorted body condition, the mean accuracy in the upright trials was $76.3 \pm 1.4 \%, 95 \% \mathrm{CI}[56.6,89.3]$, and the mean accuracy in the inverted trials was $72.4 \pm$ $1.6 \%, 95 \%$ CI $[51.4,87.1]$ (Figure 5).

457 The GLMM analyses showed that accuracy was significantly higher in the upright trials 458 than in the inverted trials in the intact condition (estimate of the fixed effect orientation: 0.44, 459 standard error: $0.10, z$ value $=4.33, p<.001$; estimate of the fixed effect condition: 0.01 ; 460 estimate of the intercept: 0.98 ) and also in the distorted condition (estimate of the fixed effect 461 orientation: 0.21 , standard error: $0.10, z$ value $=2.14, p=.03$; estimate of the fixed effect 462 condition: -0.01 ; estimate of the intercept: 0.98 ). 


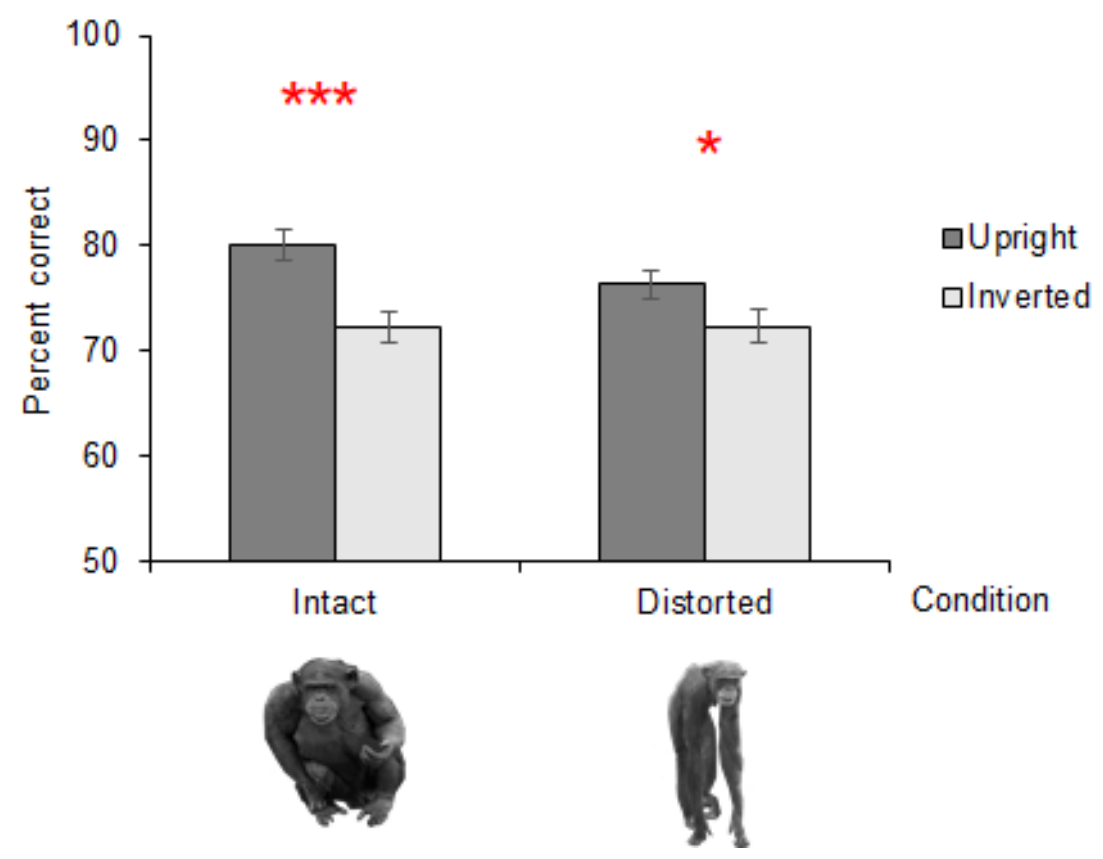

Figure 5. Chimpanzees' performances in Experiment 2. The conditions were intact

464 bodies (control) and distorted bodies (with abnormal body part proportions). Error bar: SEM;

$* p<.05 ; * * * p<.001$

466

467

Response time

No inversion effect was shown in the response-time data in the intact $(p=.33)$ or

469 distorted body condition $(p=.47)$. No interaction was found between condition and orientation

$470 \quad(p=.23)$. ms, 95\% CI [776, 1099], and the mean response time in the inverted trials was $939 \pm 22 \mathrm{~ms}$, $95 \%$ CI $[788,1123]$. In the distorted body condition, the mean response time in the upright

474 trials was $949 \pm 21 \mathrm{~ms}, 95 \%$ CI $[795,1138]$, and the mean response time in the inverted trials 
0.001 , standard error: $<0.001, t$ value $=-0.73, p=.47$; estimate of the fixed effect condition:

$<0.001$; estimate of the intercept: 0.001$)$.

\section{Discussion}

Chimpanzees showed the inversion effect in both distorted and intact body conditions.

The violation of body proportions did not make the inversion effect disappear. This finding reveals that the body configural processing in chimpanzees does not rely on normal body proportions. It also suggests that body proportions might not matter as much to chimpanzees as the first-order relations in the body configuration prototype.

In the control condition of intact bodies, we included blurring parts that inevitably occurred in abnormal bodies when making the stimuli, although the structure and proportion

490 formed normal bodies. The inversion effect remained, suggesting that chimpanzees do not pay

491 attention to the strange small local parts when they process bodies, and that the results for the manipulated bodies are not affected by the local marks that appear in making the stimuli.

\section{General discussion}

In this study, we investigated how changes in body structures influence the body configural processing of chimpanzees. We tested the inversion effect in matching-to-sample tasks. In Experiment 1, we used chimpanzee bodies with abnormal first-order relations, i.e. 
497 body part arrangements. The chimpanzee participants did not show the inversion effect in this

498 condition, suggesting that their configural body processing relies on correct first-order relations.

499 In Experiment 2, where we used chimpanzee bodies with abnormal body proportions, they also

500 showed the inversion effect. These findings suggest that the violation of body proportions does

501 not interfere with the configural processing of bodies. In both experiments, the control

502 conditions were normal chimpanzee bodies, to which they showed the inversion effect. The

503 results also implied that the first-order relations are probably important for the body

504 configuration prototype in chimpanzees' mind, while body proportions may not be.

The changes of first-order relations interfering with configural processing is the same

506 as what happens in humans (Gliga \& Dehaene-Lambertz, 2005; Reed, Stone, Grubb, \&

507 McGoldrick, 2006; Yovel, Pelc, Tubetzky, 2010) and capuchin monkeys when they recognize

508 human bodies (Matsuno \& Fujita, 2018). At least this aspect of body processing is conservative

509 in evolution. Chimpanzees, humans and capuchin monkeys rely on correct body part

510 arrangements to process bodies configurally. This finding provides implications on how

511 configural processing is shaped by experience and evolution. From the ontogenetic perspective,

512 the experience of seeing bodies with normal structures may facilitate the specific processing,

513 and a violation of normal structures would diminish the configural processing. However, this

514 specific body processing and its property regarding first-order relations must have been

515 developed during the process of evolution. From the phylogenetic perspective, the common

516 ancestors of these species may already have developed this special processing for biologically

517 important objects, bodies, and the processing may be based on correct structures. 
differently than other objects, and provide information about object processing in general. In the three aspects (sensitivity to first-order relations, second-order relations, and holistic processing) of face configural processing reviewed by Maurer and colleagues (2002), we did not focus on holistic processing of bodies in this study. The changes of first-order relations in the body interfering with configural processing is consistent with the findings in studies of face recognition, where the inversion effect was diminished by scrambling facial parts (e.g. Bentin, regarded as potentially corresponding with the second-order relation, are actually different from it (Reed, Stone, Grubb, \& McGoldrick, 2006; Zieber, Bhatt, Hayden, Kangas, Collins, \& Bada, 2010; Zieber, Kangas, Hock, \& Bhatt, 2015). The second-order relations in faces refers to the space between the first-order relational features, i.e. facial parts. However, bodies and

530 faces are different, although they share many common properties. Faces all have the same 531 contour, while the shape of body contours changes with different postures. On faces, the 532 distinguishing parts are separated from each other, but body parts are connected. Therefore, it 533 is difficult to transfer the content of the second-order relations of faces directly to bodies and 534 to compare the properties in terms of processing the second-order relation in faces and bodies 535 directly. In Experiment 2, we used intact bodies with local blurring in the control condition, 536 and chimpanzees showed the inversion effect regardless of it. This finding implies that the local 537 features do not influence configural processing. This is the same in face processing (e.g. 538 Donnelly, Humphreys, \& Sawyer, 1994). Researchers replaced the facial parts with objects, 
and still found an inversion-effect like effect.

541 configural processing, but at the same time, the detailed mechanisms might be different (Reed,

542 Stone, Grubb, \& McGoldrick, 2006; Slaughter, Stone, \& Reed, 2004; Yovel, Pelc, Lubetzky,

543 2010). This proposal is supported by fMRI studies finding distinct brain areas activated by

544 body and face processing, although in certain conditions they are adjacent and partially overlapping (Peelen \& Downing, 2005, 2007; Popivanov, Jastorff, Vanduffel, \& Vogels, 2012;

546 Premereur, Taubert, Janssen, Vogels, \& Vanduffel, 2016). Also, as discussed previously, the

547 properties of shapes are actually different between faces and bodies. However, the above

548 findings were all from human participants. It is not clear how these processes differ in other

549 species. In answering how different the processing of bodies and faces are, this study provided evidence in humans' closest relatives, chimpanzees. Future studies could include more species and adopt more diverse paradigms to examine other aspects of configural processing, for example, the holistic processing or more unique processing of bodies. The fact that humans and nonhuman animals can be trained to use configural processing (Diamond \& Carey, 1986;

554 Gauthier, Skudlarski, Gore, \& Anderson, 2000; Gauthier \& Tarr, 1997; Gauthier, Williams, Tarr,

555 \& Tanaka, 1998; Hagen \& Tanaka, 2019) suggests that this mode of processing may support 556 quick detection of things that need expertise to deal with, including bodies and faces. Research

557 from different perspectives in various species could determine if and how different factors tune object processing in different ways. 
560 show the inversion effect to intact chimpanzee bodies, and not to other objects. It is reasonable

561 to infer that if they do not show the inversion effect to certain stimuli, they might not see those

562 stimuli as the same as intact bodies. Therefore, the inversion effect might be an index of their

563 understanding of body structures in recognizing bodies with abnormal structures. However, the

564 testing paradigm used in this study cannot directly evaluate discrimination between intact and

565 abnormal bodies, so this inference is premature. Still, if we follow this logic, the conclusion

566 would be that chimpanzees are sensitive to first-order relations but not to body proportions.

567 The body prototype in their mind must have correct body part arrangements, but not necessarily

568 normal proportions. From the ecological perspective, a serious violation in the first-order

569 relations may indicate a major injury: loss of a body part or a twisted joint causing weird or

570 impossible postures. Chimpanzees have been shown to be sensitive to certain injuries (Sato,

571 Hirata, \& Kano, 2019). For body proportions, individuals actually vary in their body

572 proportions; therefore, chimpanzees would use the same strategy to process the distorted bodies

573 in Experiment 2 as they use for normal bodies, although their proportions deviated from the

574 normal level to some extent.

575 Previous studies have found that 3.5-month-old human infants could discriminate

576 between intact and abnormal bodies, both in terms of first-order relations and body proportions.

577 The insensitivity of chimpanzees to body proportions in our experiments could reflect different

578 experimental design from those used with human infants, or a true difference between humans

579 and chimpanzees in this perceptual domain. The difference could arise from differences in

580 ability to summarize body samples and to build an average body figure with structures in the 
581 normal range. However, chimpanzees showed comparable performances as humans in a task

582 to choose the average sizes of objects (Imura, Kawakami, Shirai, \& Tomonaga, 2017),

583 suggesting that they are able to make summaries and averages. The groups in our study were

584 composed of 12 or 13 individuals. Perhaps their limited experience of conspecifics was not

585 enough for the chimpanzees to build pictures of a normal range of body proportions, although

586 it was apparently enough for them to have a clear understanding of body part arrangements.

587 The latter is fixed, but the former can vary across individuals. For future directions, more direct

588 paradigms could be adopted to test chimpanzees' understanding of body structures, including

589 discrimination tasks that were used in infants (Zieber, Bhatt, Hayden, Kangas, Collins, \& Bada,

590 2010; Zieber, Kangas, Hock, \& Bhatt, 2015). Other techniques, for example, eye tracking,

591 could be used to examine their attention to (abnormal) body structures in a more direct and

592 probably more detailed way (Kano \& Tomonaga, 2009).

593 In summary, this study shows that chimpanzees' configural processing for bodies relies

594 on correct first-order relations, but the body proportion does not matter. The results also imply

595 that chimpanzees may be more sensitive to first-order relations than body proportions in their

596 understanding of body structures.

597

598

599

600

601 
603 Bates, D., Mächler, M., Bolker, B., \& Walker, S. (2015). Fitting Linear Mixed-Effects

604 Models Using lme4. Journal of Statistical Software, 67(1), 1-48.

605 Bentin, S., Allison, T., Puce, A., Perez, E., \& McCarthy, G. (1996). Electrophysiological 606 studies of face perception in humans. Journal of cognitive neuroscience, 8(6), 551-

607 565.

608

Carey, S. (1992). Becoming a face expert. Phil. Trans. R. Soc. Lond. B, 335(1273), 95-103.

609

Dahl, C. D., Rasch, M. J., Tomonaga, M., \& Adachi, I. (2013). The face inversion effect in

610 non-human primates revisited-an investigation in chimpanzees (Pan

611 troglodytes). Scientific reports, 3, 2504.

612 Diamond, R., \& Carey, S. (1986). Why faces are and are not special: an effect of expertise. Journal of Experimental Psychology: General, 115(2), 107.

614 Donnelly, N., Humphreys, G. W., \& Sawyer, J. (1994). Stimulus factors affecting the 615 categorisation of faces and scrambled faces. Acta Psychologica, 85(3), 219-234.

616 Gao, J., \& Tomonaga, M. (2018). The body inversion effect in chimpanzees (Pan troglodytes). PloS one, 13(10), e0204131.

618 Gauthier, I., \& Tarr, M. J. (1997). Becoming a "Greeble" expert: Exploring mechanisms for 619 face recognition. Vision research, 37(12), 1673-1682.

620 Gauthier, I., Skudlarski, P., Gore, J. C., \& Anderson, A. W. (2000). Expertise for cars and birds recruits brain areas involved in face recognition. Nature Neuroscience, 3(2), 191-197. doi:10.1038/72140 
623 Gauthier, I., Williams, P., Tarr, M. J., \& Tanaka, J. (1998). Training 'greeble'experts: a framework for studying expert object recognition processes. Vision research, 38(15-16),

625 2401-2428.

626

Gliga, T., \& Dehaene-Lambertz, G. (2005). Structural encoding of body and face in human

627 infants and adults. Journal of Cognitive Neuroscience, 17(8), 1328-1340.

628

Hagen, S., \& Tanaka, J. W. (2019). Examining the neural correlates of within-category discrimination in face and non-face expert recognition. Neuropsychologia, 124, 44-54.

630 doi:10.1016/j.neuropsychologia.2019.01.005

631

Haig, N. D. (1984). The effect of feature displacement on face recognition. Perception, 13(5),

632 505-512.

633

Hirata, S., Hirai, H., Nogami, E., Morimura, N., \& Udono, T. (2017). Chimpanzee Down syndrome: a case study of trisomy 22 in a captive chimpanzee. Primates, 58(2), 267-

635 273.

636

637

Hobaiter, C., \& Byrne, R. W. (2014). The meanings of chimpanzee gestures. Current Biology, 24(14), 1596-1600.

638

Imura, T., Kawakami, F., Shirai, N., \& Tomonaga, M. (2017). Perception of the average size of 639 multiple objects in chimpanzees (Pan troglodytes). Proceedings of the Royal Society B:

640 Biological Sciences, 284(1861), 20170564.

641 Kano, F., \& Tomonaga, M. (2009). How chimpanzees look at pictures: a comparative eye642 tracking study. Proceedings of the Royal Society of London B: Biological Sciences, rspb-2008. 
644 Le Grand, R., Mondloch, C. J., Maurer, D., \& Brent, H. P. (2001). Neuroperception: Early 645 visual experience and face processing. Nature, 410(6831), 890.

646 Martinez, L., \& Matsuzawa, T. (2009). Auditory-visual intermodal matching based on 647 individual recognition in a chimpanzee (Pan troglodytes). Animal cognition, 12(1),

648 $71-85$.

649 Matsumoto, T., Itoh, N., Inoue, S., \& Nakamura, M. (2016). An observation of a severely 650 disabled infant chimpanzee in the wild and her interactions with her

651 mother. Primates, 57(1), 3-7.

652

Matsuno, T., \& Fujita, K. (2018). Body inversion effect in monkeys. PloS one, 13(10),

653 e0204353.

654

655

Matsuzawa, T., Humle, T., \& Sugiyama, Y. (Eds.). (2011). The chimpanzees of Bossou and Nimba. Springer Science \& Business Media.

Matsuzawa, T., Tomonaga, M., \& Tanaka, M. (Eds.). (2006). Development in chimpanzees.

657 Tokyo: Springer.

658

Maurer, D., Le Grand, R., \& Mondloch, C. J. (2002). The many faces of configural processing. Trends in cognitive sciences, 6(6), 255-260.

660 Parr, L. A., Dove, T., \& Hopkins, W. D. (1998). Why faces may be special: evidence of the 661 inversion effect in chimpanzees. Journal of Cognitive Neuroscience, 10(5), 615-622.

662 Peelen, M. V., \& Downing, P. E. (2005). Selectivity for the human body in the fusiform gyrus. Journal of neurophysiology, 93(1), 603-608. 
664 Peelen, M. V., \& Downing, P. E. (2007). The neural basis of visual body perception. Nature

665 Reviews Neuroscience, 8(8), 636.

666

Popivanov, I. D., Jastorff, J., Vanduffel, W., \& Vogels, R. (2012). Stimulus representations in

667 body-selective regions of the macaque cortex assessed with event-related

668 fMRI. Neuroimage, 63(2), 723-741.

669

Premereur, E., Taubert, J., Janssen, P., Vogels, R., \& Vanduffel, W. (2016). Effective

670 connectivity reveals largely independent parallel networks of face and body

671 patches. Current Biology, 26(24), 3269-3279.

672

Quinn, P. C., Lee, K., Pascalis, O., \& Slater, A. M. (2007). In support of an expert-novice difference in the representation of humans versus non-human animals by infants: generalization from persons to cats occurs only with upright whole images. Cognitie, Creier, Comportament/Cognition, Brain, Behavior, 11(4).

R Core Team. (2017). R: A language and environment for statistical computing. Vienna, Austria: R Foundation for Statistical Computing.

Ramm, B. J., Cummins, T. D., \& Slaughter, V. (2010). Specifying the human body configuration. Visual Cognition, 18(6), 898-919.

680 Reed, C. L., Stone, V. E., Bozova, S., \& Tanaka, J. (2003). The body-inversion 681 effect. Psychological science, 14(4), 302-308.

682 Reed, C. L., Stone, V. E., Grubb, J. D., \& McGoldrick, J. E. (2006). Turning configural 683 processing upside down: part and whole body postures. Journal of Experimental Psychology: Human Perception and Performance, 32(1), 73. 
685 Robbins, R. A., \& Coltheart, M. (2012). The effects of inversion and familiarity on face 686 versus body cues to person recognition. Journal of Experimental Psychology: Human 687 Perception and Performance, 38(5), 1098.

688

Sato, Y., Hirata, S., \& Kano, F. (2019). Spontaneous attention and psycho-physiological 689 responses to others' injury in chimpanzees. Animal cognition, 1-17.

690

Slaughter, V., Stone, V. E., \& Reed, C. (2004). Perception of faces and bodies: Similar or

691 different?. Current directions in psychological science, 13(6), 219-223.

692

Tanaka, J. W., \& Farah, M. J. (1993). Parts and wholes in face recognition. The Quarterly 693 Journal of Experimental Psychology Section A, 46(2), 225-245.

694

Tomonaga, M. (1999). Inversion effect in perception of human faces in a chimpanzee (Pan

695 troglodytes). Primates, 40(3), 417-438.

696 Tomonaga, M., \& Imura, T. (2015). Efficient search for a face by chimpanzees (Pan troglodytes). Scientific reports, 5, 11437.

698 Tomonaga, M., Itakura, S., \& Matsuzawa, T. (1993). Superiority of conspecific faces and reduced inversion effect in face perception by a chimpanzee. Folia 141.

Yovel, G., Pelc, T., \& Lubetzky, I. (2010). It's all in your head: why is the body inversion effect abolished for headless bodies?. Journal of Experimental Psychology: Human Perception and Performance, 36(3), 759. 
706 Zieber, N., Bhatt, R. S., Hayden, A., Kangas, A., Collins, R., \& Bada, H. (2010). Body

707 representation in the first year of life. Infancy, 15(5), 534-544.

708 Zieber, N., Kangas, A., Hock, A., \& Bhatt, R. S. (2015). Body structure perception in infancy.

709 Infancy, 20(1), 1-17.

710

711 Supplementary Materials

712 Data file S1 Accuracy and response-time data in each condition 